\title{
DETERMINAÇÃO DO TEOR DE PARACETAMOL EM MEDICAMENTOS GENÉRICOS PELA TÉCNICA DE ESPECTROFOTOMETRIA UV.
}

DETERMINATION OF PARACETAMOL IN GENERIC DRUGS BY UV SPECTROPHOTOMETRY.

Gilmar Aires da Silva

Docente do Instituto Federal de Goiás Campus Uruaçu. gilmaraires@hotmail.com

\section{Luciano Alves da Silva}

Docente do Instituto Federal de Goiás Campus Uruaçu.

luciano tj@yahoo.com.br

\section{Daniella Cintra Hilário}

Discente do Curso Técnico em Química do Instituto Federal de Goiás Campus Uruaçu. daniella-cintra-hilário@ hotmail.com

\section{Paula Micaelly Ferreira Bueno}

Discente do Curso Técnico em Química do Instituto Federal de Goiás Campus Uruaçu. pcdapaula@hotmail.com

\section{RESUMO:}

Introdução: Atualmente, com os processos de industrialização e a vida mecanizada, a implantação de processos que visam à qualidade é essencial. No Brasil, a Agência Nacional de Vigilância Sanitária (ANVISA) e a Farmacopeia Brasileira (FB), procuram estabelecer, a partir de normativas, padrões necessários para a obtenção de um produto de boa validação. A preocupação torna-se ainda maior no setor de medicamentos, pois este está diretamente relacionado com questões éticas, legais e essencialmente, a saúde pública. Devido a este fato é imprescindível que sejam adotadas algumas políticas, como a adoção da Gestão da Qualidade farmacêutica e ferramentas do controle de qualidade. O paracetamol é um medicamento genérico altamente comercializado. Objetivo: Comparar a quantidade de princípio ativo no medicamento genérico em relação a quantidade no medicamento referência. Metodologia: Por meio da técnica de espectrofotometria pode-se determinar a quantidade de substância ativa no medicamento verificando se os fabricantes do genérico paracetamol estão cumprindo os requisitos propostos pela FB no que se refere ao Controle de Qualidade Físico-Químico utilizando a técnica de espectrometria UV. Resultados: Os resultados obtidos evidenciam a eficácia da técnica utilizada. Conclusão: A pesquisa realizada demonstrou que os lotes do medicamento paracetamol em análise estão em conformidade com o medicamento genérico. 
Palavras chaves: Paracetamol, Espectrometria UV, Medicamento Genérico e Referência.

\begin{abstract}
:
Introduction: Currently, with the processes of industrialization and mechanized life, the implementation of processes that aim to quality is essential. In Brazil, the National Health Surveillance Agency ( ANVISA ) and the Brazilian Pharmacopoeia ( FB ), seek to establish, from regulations, standards necessary to obtain a product of good validation. The concern becomes even greater in the drug industry, as this is directly related to ethical, legal and essentially public health. Due to this fact it is essential that some policies are adopted, as the adoption of the management of the pharmaceutical quality and quality control tools. Paracetamol is a highly commercialized generic drug. Objective: To compare the amount of active ingredient in the generic medicinal product for the amount in the reference product. Methodology: Using the spectrophotometric technique can determine the amount of active substance in the medicament checking whether the generic paracetamol manufacturers are meeting the requirements proposed by the FB with respect to the Physical -Chemical Quality control using UV spectrometry. Results: The results show the effectiveness of the technique.
\end{abstract} Conclusion: The research showed that lots of drug acetaminophen in analysis are in accordance with the generic drug.

Keywords: Paracetamol, UV Spectrometry, Generic Drug and Reference.

Endereço de Correspondência:

Instituto Federal de Goiás - Campus Uruaçu

Rua Formosa, Qd. 28/29, Loteamento Santana. CEP: 76400-000. Uruaçu - GO

Fone: (62)3357-8150

\title{
INTRODUÇÃO
}

Desde a antiguidade o homem se preocupa com a qualidade de seus produtos. A qualidade pode ser definida como um conjunto de atributos que se deseja para um determinado produto. Com o passar dos anos, esta evoluiu ao ponto que, hoje se podem prevenir os defeitos desde a etapa do projeto até o produto acabado. Isto permite garantia de maior qualidade dos produtos (GIL; QUINTINO, 2010). O controle de qualidade é essencial durante todas as fases de processamento, englobando diversos métodos que favorecem a manutenção de um resultado ideal (JUNG, 2008).

A Agência Nacional de Vigilância Sanitária (ANVISA) é o órgão federal que fiscaliza a aplicação do controle de qualidade nas indústrias brasileiras. Especificamente em laboratórios de análises físico-químicas, são seguidas regulamentações advindas da 
Farmacopeia Brasileira, a qual é considerada o código oficial farmacêutico do país (LIMA, 2012). Quanto ao controle de qualidade dos medicamentos deve-se ser muito mais criterioso quanto aos resultados, visto que envolve questões éticas e legais, estando diretamente ligada a saúde pública (GIL; GONÇALVES; FIGUEIREDO, 2010). Sendo assim, o controle de qualidade de medicamentos possui a finalidade de garantir a pureza, eficácia e segurança àqueles que fazem uso dos fármacos (BRASIL, 2009).

Segundo a Resolução da Diretoria Colegiada nº17 (RDC) de 16 de abril de 2010, todo fabricante de medicamentos deve possuir um controle de qualidade que, deve ser separado da linha de produção e dos demais departamentos envolvidos na fabricação. Dentro do departamento de controle de qualidade utilizam-se ferramentas como as Boas Práticas de Fabricação (BPF) que permitem que o fármaco não seja liberado para comercialização antes de ter a eficácia garantida (BRASIL, 2010).

Os laboratórios de controle de qualidade físico-químicos devem ter implantados uma política de Gestão da Qualidade Farmacêutica proveniente da necessidade de assegurar a conformidade com a legislação. São também utilizados testes com o fim de proporcionar a validação dos medicamentos fabricados nas indústrias, visando garantir a segurança do futuro consumidor do mesmo (RODRIGUES, et.al, 2014).

Dentre os tipos de medicamento documentados, há os de referência e os genéricos, que são consumidos em larga escala. De acordo com a Lei 9787/99, medicamento de referência é um produto inovador no qual o fabricante desenvolveu a formulação e a forma farmacêutica adequada à via de administração, bem como o interesse terapêutico, estabelecendo e validando os processos de fabricação. Além disso, este foi registrado na ANVISA, a qual comprovou cientificamente a segurança e qualidade do produto a ser comercializado no país (BRASIL, 1999).

Quanto aos medicamentos genéricos, são produtos semelhantes aos de referência que devem cumprir com as especificações existentes, conferindo a mesma bioequivalência e biodisponibilidade certificadas pela ANVISA. O teste de equivalência é feito por meio de testes físicos e físico-químicos que comparam o medicamento candidato a genérico e o seu respectivo medicamento de referência. Entretanto, é aceitável que o referido não tenha a mesma formulação e processo de fabricação, desde que não comprometam a equivalência farmacêutica. Além disso, os medicamentos 
genéricos só podem ser produzidos e comercializados quando há quebra de patente (BRASIL, 1999).

A Farmacopeia Brasileira (FB) é o código oficial do Brasil, que estabelece os principais padrões a serem adotados, no que se refere às análises físico-químicas. Em sua $\mathrm{V}$ edição estabeleceu que o medicamento paracetamol em compridos, deve obter em sua composição no mínimo $95,0 \%$ e no máximo $105,0 \%$ de princípio ativo. A identificação é feita a partir de testes, os quais em conjunto formulam as etapas para uma boa qualidade do fármaco (ANVISA, 2010).

O teste de doseamento envolve a determinação da quantidade de substância ativa no fármaco. A partir da metodologia deste teste, a solução pode ser disposta a análises de identificação espectrométricas, cujas podem ser: ultravioleta (UV), vísivel (VIS), infravermelho médio (MIR) e infravermelho próximo (NIR). A utilização de técnicas a partir da luz dispõe de padrões pré-estabelecidos por normativas, possuindo um caráter especifico para cada tipo de espécie química (BECKTOLD,2008).

As técnicas utilizadas para soluções com concentração na ordem de $1 \mu \mathrm{g} / \mathrm{mL}$ incluem a UV e VIS, sendo que MIR e NIR compreendem substâncias de concentração de $100 \mathrm{mg} / \mathrm{mL}$ (SIMCH, 2013). A FB estabelece que a determinação da concentração do analito a partir da absorção molecular UV ou VIS, utiliza de uma fonte principal, que é uma onda eletromagnética, em um comprimento de onda específico e esta incide sobre a solução contendo o principio ativo, expondo a absorbância da diluição. A técnica está diretamente relacionada à lei de Lambert-Beer. Assim, cabe destacar que o medicamento paracetamol deve ser analisado em um espectro de luz situado em 257 nm, fazendo ligação ao espectro UV (FARMACOPEIA BRASILEIRA, 2010).

O objeto deste trabalho consiste em comparar a quantidade de princípio ativo no medicamento genérico em relação à quantidade no medicamento referência, assim verificar se os fabricantes do medicamento paracetamol (referência e genérico) estão cumprindo os requisitos mínimos propostos pela Farmacopeia Brasileira no que se refere ao Controle de Qualidade Físico- Químico.

\section{Materiais e métodos}

Amostras Tylenol ${ }^{\circledR}$

Amostras de paracetamol comercial. 
Balança analítica SHIMADZU - MODELO AUY 220

Balões volumétricos de $25 \mathrm{~mL}, 100 \mathrm{~mL}$ e $200 \mathrm{~mL}$

Cubeta de quartzo 3,5 mL, caminho óptico de $1 \mathrm{~cm}$

Espectrofotômetro UV SPECTRUM - MODELO 2000 UV

Micropipeta de $100 \mu \mathrm{L}$ a $1000 \mu \mathrm{L}$

Pipetas de Pasteur

Solução de hidróxido de sódio $(\mathrm{NaOH})$ Merck 0,1M e 0,01M

Vidrarias em geral

O doseamento é utilizado para a determinação da quantidade de princípio ativo que está presente no fármaco. Para se realizar este teste foi pesado e triturado 20 comprimidos e transferiu-se $0,15 \mathrm{~g}$ do pó obtido para um balão volumétrico de $200 \mathrm{~mL}$. Em seguida, adicionou-se $50 \mathrm{~mL}$ de hidróxido de sódio $0,1 \mathrm{M}$ e $100 \mathrm{~mL}$ de água agitando constantemente por 15 minutos completando o volume do balão com água. Parte da solução $(10 \mathrm{~mL})$ foi filtrada e diluída em $100 \mathrm{~mL}$ de água, após esse procedimento transferiu-se $10 \mathrm{~mL}$ da solução resultante para um balão volumétrico de $100 \mathrm{~mL}$ com $10 \mathrm{~mL}$ de hidróxido de sódio $0,01 \mathrm{M}$. Terminada a solução, esta foi levada ao espectrofotômetro UV onde a absorbância foi lida no comprimento de onde de 257 $\mathrm{nm}$. Todos os ensaios forma realizados em triplicata (FARMACOPEIA BRASILEIRA, 2010).

\section{Resultados e discussão}

Quanto aos medicamentos genéricos, são produtos semelhantes aos de referência que devem cumprir com as especificações existentes, conferindo a mesma bioequivalência e biodisponibilidade certificadas pela ANVISA. O teste de equivalência é feito por meio de testes físicos e físico-químicos que comparam Entretanto, é aceitável que o referido não tenha a mesma formulação e processo de fabricação, desde que não comprometam a equivalência farmacêutica.

Para comparar a semelhança entre os medicamentos de referência e genérico quanto à bioequivalência e biodisponibilidade conforme especificado pela ANVISA foi feito o teste de doseamento do princípio ativo paracetamol em triplicata. Essa comparação é importante, pois o medicamento candidato a genérico precisa conter a 
mesma quantidade de princípio ativo presente no genérico em comparação com o medicamento referência.

A absorbância obtida para o princípio ativo paracetamol referência $\left(\right.$ Tylenol $^{\circledR}$ ) foi de 0,422 $\pm 0,002$, já para o genérico obteve-se 0,418 $\pm 0,002$ de absorbância. Com estes dados determinados a partir da técnica de espectroscopia realizada na região UV, pode-se notar que os respectivos resultados tiveram uma mínima diferença no sinal analítico $(\mathrm{Abs}=0,004)$ indicando que os dois medicamentos estão com praticamente a mesma quantidade de princípio ativo. $\mathrm{O}$ conjunto de dados foi tratado estatisticamente utilizando teste de significância de t Student com 95\% de confiança. O mesmo apresentou a hipótese nula verdadeira evidenciando que não há diferença significativa no conjunto de dados. SILVEIRA E ORTIZ (2012) corroboram com estes resultados, pois em experimentos semelhantes encontraram valores compatíveis com os dados desta pesquisa.

\section{Conclusão}

O controle de qualidade em qualquer âmbito é de extrema importância, sendo que envolve fatores éticos e legais. A pesquisa realizada demonstrou que os lotes de medicamento paracetamol em análise estão de acordo com os pré-requisitos propostos pela Farmacopeia Brasileira 2010. De forma que, utilizando o medicamento de referência como padrão e confrontando este com o medicamento genérico, pode - se observar valores próximos, validando assim a conformidade do genérico.

\section{Agradecimentos:}

Ao CNPq pelo fomento do projeto e ao Instituto Federal de Goiás Campus Uruaçu por disponibilizar espaço e equipamentos para a realização dos experimentos.

\section{Referencias}

BECKTOLD, CAROLINA GIULIANE. Análise comparativa de qualidade de comprimidos de captopril: genérico, similar e de referência. Monografia] Blumenau: Universidade Regional de Blumenau, 2008.

BRASIL, Lei $\mathrm{n}^{\circ} 9.787$ de 10 de fevereiro de 1999. Altera a Lei 6.360 de 23 de setembro de 1976, que dispõe sobre a vigilância sanitária estabelece o medicamento genérico, dispõe sobre a utilização de nomes genéricos em produtos farmacêuticos. Diário Oficial da União. Brasília, 10 de fevereiro de 1999. 
BRASIL. ANVISA. Diretoria Colegiada. Resolução-RDC no 17, 16 de abril de 2010. Dispõe sobre as boas práticas de fabricação de medicamentos. Diário Oficial da União : 21 de agosto de 2006.

FARMACOPEIA BRASILEIRA. monografias. 5a ed. Brasília, 2010. v.1 e 2.

GIL, E. S.; QUINTINO, W. A. Gestão de qualidade. In: GIL, E.S. Controle físicoquímico de qualidade de medicamentos. 3. ed. São Paulo: Pharmabooks, 2010. p.2941.

JUNG, M. R. Controle de qualidade físico-químico em comprimidos de hidroclorotiazida 50mg. Novo Hamburgo, 2008. Trabalho de Conclusão de Curso. Centro Universitário Freevale, Instituto de Ciência da Saúde, curso de ciências farmacêuticas. "Disponível em": <http://ged.feevale.br/bibvirtual/Artigo/ArtigoMarcioJung.pdf $>$ Acesso em: 05/042015

LOYVD Jr. ,V. ALLEN; POPOVICH, N. G; ANSEL, H. C. Formas farmacêuticas e sistemas de liberação de fármacos. 8 ed. São Paulo: Ed. Permier , 2007.

LIMA, M., A. FISICO, AVALIAÇÃO DO CONTROLE DE QUALIDADE. Tese de Doutorado. UNIVERSIDADE ESTADUAL DE GOIÁS, 2012.

SIMCH, Fernanda Heloisa. Testes aplicados em estudos de equivalência farmacêutica (2013).

SILVEIRA, R; ORTIZ, J. Controle de qualidade em paracetamol matéria-prima. 52 ${ }^{\circ}$ Congresso Brasileiro de Química, Pernambuco, 2012. <http://www.abq.org.br/cbq/2012/trabalhos/4/920-14585.html> Acesso em 31 de março de 2015.

SCHUH, Diego Callai et al. Intoxicação e exposições por paracetamol: análise de seis anos de registros de Centro de Informações Toxicológicas de Santa Catarina-CIT/SC. 2007.

SEQUINEL, R. Desenvolvimento de novos procedimentos analíticos para a determinação de paracetamol em amostras de medicamentos. 2008. Dissertação (Mestrado em Química) - Universidade Estadual Paulista, Araraquara, São Paulo. 